\title{
No Run Gravity
}

\author{
Eric V. Linder ${ }^{1,2}$ \\ ${ }^{1}$ Berkeley Center for Cosmological Physics 85 Berkeley Lab, \\ University of California, Berkeley, CA 94720, USA \\ ${ }^{2}$ Energetic Cosmos Laboratory, Nazarbayev University, Astana, Kazakhstan 010000
}

(Dated: March 7, 2019)

\begin{abstract}
Considering the dark energy/gravity landscape if next generation surveys of galaxies, cosmic microwave background radiation, and gravitational waves do not find clear modification of gravity, we develop No Run Gravity as a counterexample to the conclusion that this would imply general relativity with an expansion history described by an equation of state $w(z)$. No Run Gravity is a cubic Horndeski theory with a constant Planck mass, no gravitational slip, and no modification of gravitational waves, but a rich phenomenology beyond $w(z)$. We calculate the evolution of gravitational strength, sound speed, and cosmic growth within the theory and project sensitivities for upcoming DESI redshift space distortion data.
\end{abstract}

\section{INTRODUCTION}

Many signatures can appear in cosmic surveys to provide evidence of gravitational properties beyond general relativity. These can include modification of the tensor sector - gravitational waves $(\mathrm{GW})$ - surveyed by laser interferometers such as LIGO/Virgo and LISA and by cosmic microwave background (CMB) B-mode polarization experiments such as Simons Observatory, CMB-S4, and LiteBIRD (see, e.g., [1, 2]. Already, deviation of GW propagation from the speed of light is effectively ruled out, at least in certain regimes [3]. Damping of the GW amplitude over the time of propagation is allowed and related to the running of the Planck mass [4-6]. Testing this through comparison of GW and electromagnetic luminosity distances to the same source will be highly interesting, especially since in certain theories it can be directly connected to suppression in the growth of cosmic structure [7].

In the scalar - density perturbation - sector, signatures include modification of the growth of large scale structure, modification of the propagation of light (lensing), and gravitational slip, where the metric gravitational potentials differ from each other, unlike in general relativity. These can be probed by large scale structure surveys, through galaxy clustering and weak gravitational lensing, such as with DESI, Euclid, LSST, and WFIRST, and CMB surveys (see, e.g., $[8,9]$ for recent reviews).

This rich array of observational effects, and the phenomenology that goes with them, is comforting, as giving rise to hope that the excellent data will reveal key clues to the nature of cosmic acceleration and gravity. Suppose, however, that the data does not show significant deviation from general relativity - no change in GW speed or damping, no gravitational slip. Change in the expansion history or growth history can be accommodated within general relativity by modification of the effective dark energy equation of state $w(z)$ from the concordance value $w=-1$ of the cosmological constant. Would such observations then imply the universe is described solely by general relativity with some $w(z)$ ?

Such a question has been partially addressed by the introduction of No Slip Gravity [7], where a modified gravity theory was defined that had no change in GW speed or gravitational slip. This does have damping of GW amplitudes, however, as it involves a running Planck mass. Here we go one step further and consider no slip plus no running: No Run Gravity. This will give no change in the tensor sector (and so indeed may not be considered true modified gravity), and the two metric potentials will be the same.

However - the metric potentials do not have to be equal to Newton's constant, and so there can remain effects on cosmic growth and on light deflection in a manner distinct from any $w(z)$ (and indeed simple scalar field generalizations like k-essence $[10,11])$. Thus we will continue to refer to it as a modified gravity theory in an informal way.

No Run Gravity can be viewed as a minimal modification to general relativity. The hope is that it will serve as a benchmark to test deviations from general relativity and show the science reach of next generation surveys even when the more dramatic signatures may not be found.

In Sec. II we set up the theory and relate it to Horndeski gravity and effective field theory. Section III discusses the implications of simple parametrizations, including stability and early and late time limits. We project constraints on the theory from future data in Sec. IV and summarize and conclude in Sec. V.

\section{THEORY OF NO RUN GRAVITY}

One can approach modified gravity theories through an effective field theory or property function approach, or the Horndeski Lagrangian for the most general scalartensor theory with second order equations of motion. The equivalence between these is given in detail in [12]. The property function approach has the advantage of being able to state the physical conditions succinctly: for GW speed to equal the speed of light then the property function $\alpha_{T}=0$, and for no running of the Planck mass then the property function $\alpha_{M}=0$. In fact, we want the 
more physical condition of vanishing slip. No Slip Gravity achieves this through $\alpha_{B}=-2 \alpha_{M}$, but an appendix in [7] (also see [13]), briefly discussed an alternate method of accomplishing this by setting $\alpha_{M}=0$. We therefore define No Run Gravity as $\alpha_{M}=0=\alpha_{T}$.

This leaves the braiding property function $\alpha_{B}$, and the observationally mostly moot kineticity $\alpha_{K}[12,14]$, as well as the expansion history in terms of the Hubble parameter $H(z)$ or effective dark energy equation of state $w(z)$. In terms of the Horndeski Lagrangian the theory is

$$
\mathcal{L}=\frac{1}{2} R+K(\phi, X)-G(\phi, X) \square \phi
$$

where in the cosmic background $X=(1 / 2)(\dot{\phi})^{2}$. This relates to the full Horndeski Lagrangian by setting $G_{5}=$ $0, G_{4}=1 / 2$, and $G_{3}=G(\phi, x)$. Such a Lagrangian has been used in kinetic gravity braiding dark energy [15], inflation [16], and to solve the original cosmological constant problem [17].

Following [12] we can write the property functions as

$$
\begin{aligned}
\alpha_{B}= & \frac{2 \dot{\phi} X}{H} G_{X} \\
\alpha_{K}= & \frac{12 \dot{\phi} X}{H}\left(G_{X}+X G_{X X}\right) \\
& +\frac{2 X}{H^{2}}\left(K_{X}+2 X K_{X X}-2 G_{\phi}-2 X G_{\phi X}\right),
\end{aligned}
$$

where subscripts $\phi$ and $X$ denote derivatives with respect to that variable. Note that a k-essence scalar field model, while having a sound speed degree of freedom, achieves this through the $K$ function; it has no $G$ function and so its $\alpha_{B}=0$.

In the early universe, when $H \gg 1$ (normalizing it by its value today), we might expect $\alpha_{K} \propto \alpha_{B}$. Similarly one can show that the effective dark energy density $\rho_{\mathrm{de}} \sim H \dot{\phi} X G_{X}$ under these circumstances so $\alpha_{i} \propto$ $\Omega_{\mathrm{de}}(a)$, where $\Omega_{\mathrm{de}}(a) \sim \rho_{\mathrm{de}} / H^{2}$ is the dark energy density in units of the critical density. As very strongly cautioned by [18] this relies on several assumptions, with the key ones being that a single Lagrangian function, e.g. $G_{X}$, dominates and that $H \gg 1$; note [18] emphasizes these hold for at best $z>10$ unless there is fine tuning, so well outside the range of most observational data. One can show that the theory can be ghost free and stable if $G_{X} \sim X^{n \geq-1}$.

We choose to work with a shift symmetric theory, so we take $K_{\phi}=0, G_{\phi}=0$. In general we will take the background expansion to be given by $\Lambda$ CDM.

\section{GRAVITY AND GROWTH EVOLUTION}

As mentioned, while No Run Gravity does not alter the tensor sector, and (by construction) has no difference between the two (time-time and space-space) metric potentials, hence no gravitational slip, it does have a modification in the strength of gravity. This is conventionally written in terms of the modified Poisson equations for nonrelativistic (matter) and relativistic (lightlike) particles, and referred to as $G_{\text {matter }}$ and $G_{\text {light }}$.

No Run Gravity has

$$
\begin{aligned}
G_{\text {eff }} & \equiv G_{\text {matter }}=G_{\text {light }}=\frac{\alpha_{B}+\alpha_{B}^{\prime}}{\alpha_{B}\left(1-\alpha_{B} / 2\right)+\alpha_{B}^{\prime}} \\
& =1+\frac{\alpha_{B}^{2}}{\alpha_{B}\left(2-\alpha_{B}\right)+2 \alpha_{B}^{\prime}},
\end{aligned}
$$

where we have normalized by Newton's constant so general relativity has $G_{\text {matter }}=G_{\text {light }}=1$. A prime denotes $d / d \ln a$, where $a$ is the cosmic scale factor. We can rewrite this as a differential equation for $\alpha_{B}$ :

$$
\alpha_{B}^{\prime}=\alpha_{B}\left[-1+\frac{\alpha_{B}}{2} \frac{G_{\mathrm{eff}}}{G_{\mathrm{eff}}-1}\right] \text {. }
$$

Thus we can either specify $G_{\text {eff }}(a)$ and solve for $\alpha_{B}$ from Eq. (6), or specify $\alpha_{B}(a)$ and determine $G_{\text {eff }}$ from Eq. (4). To satisfy early universe constraints that gravity should look like general relativity for primordial nucleosynthesis and CMB last scattering, we want both $\alpha_{B}$ and $G_{\text {eff }}-1$ to vanish at early times, $a \ll 1$. Suppose they evolve together such that $\alpha_{B} /\left(G_{\text {eff }}-1\right)=k$, with $k$ constant. This, together with $\alpha_{B} \ll 1$ at these early times, implies $\alpha_{B} \propto\left(G_{\text {eff }}-1\right) \propto a^{-1+k / 2}$. If we wanted $\alpha_{B} \propto \Omega_{\mathrm{de}}(a)$ at early times, this implies (for a $\Lambda \mathrm{CDM}$ background) $k=8$. We emphasize though that this is only reasonable for very early times.

The form for $\alpha_{B}$ can be quite varied, within stability considerations. One could also prefer to choose $G_{\text {eff }}(a)$ instead and derive $\alpha_{B}$. In either case, we expect general relativity at early times $\left(G_{\text {eff }}=1, \alpha_{B}=0\right)$ and a frozen, constant value in a de Sitter future. The simplest, and most tractable, reasonable form would be the e-fold / $1+$ tanh form used in [7]. In this case,

$$
\alpha_{B}(a)=\frac{A}{1+e^{-\left(\ln a-\ln a_{t}\right) / \tau}}=\frac{A}{1+\left(a / a_{t}\right)^{-1 / \tau}},
$$

where $A$ is the amplitude of the transition (and maximum value of $\alpha_{B}$; recall from [7] that stability requires $\left.\alpha_{B} \geq 0\right), a_{t}$ is the location of the transition, and $\tau$ is the sharpness of the transition in e-folds.

An especially nice aspect of this form is that it corresponds to the identical functional form in $G_{\text {eff }}-1$. That is,

$$
G_{\mathrm{eff}}(a)=1+\frac{A_{G}}{1+\left(a / a_{t, G}\right)^{-1 / \tau}}
$$

where

$$
\begin{aligned}
A_{G} & =\frac{A}{2-A}, \\
a_{t, G} & =a_{t}\left(\frac{1+1 / \tau}{1-A / 2}\right)^{\tau} .
\end{aligned}
$$

If one forced $\alpha_{B} \propto \Omega_{\mathrm{de}}(a)$ even beyond early times (which has no physical justification), this can be handled 
by this form with $\tau=1 / 3$ and $a_{t}=\left[\Omega_{m} /\left(1-\Omega_{m}\right)\right]^{1 / 3} \approx$ 0.75 , where $\Omega_{m}$ is the present dimensionless matter density $\Omega_{m}=1-\Omega_{\mathrm{de}}(a=1)$, which is a rather late transition (i.e. $\alpha_{B}$ is halfway through its transition at $z=0.33$, and $G_{\text {eff }}$ not until $z=-0.24$ in the future). However, the efold form is considerably more flexible.

From Eq. (6) we can see that arbitrary assumed functional forms can easily fail. Looking at the term $\alpha_{B} /\left(G_{\text {eff }}-1\right)$, we see that if $\alpha_{B}$ doesn't "keep up" with $G_{\text {eff }}-1$ (even due to numerical noise in the nonlinear equation) then the other, -1 term is likely to dominate and $\alpha_{B}^{\prime}$ will be driven negative. This implies that $\alpha_{B}$ retreats to zero, and by Eq. (4), $G_{\text {eff }}$ is driven to one. That is, deviations from general relativity will not be described successfully. Conversely, if $\alpha_{B}$ grows too fast then $\alpha_{B}^{\prime}$ gets large and there is a runaway process that violates the stability condition. Note that we do not want $\alpha_{B} \propto\left(G_{\text {eff }}-1\right)$ in general; this too can runaway. Rather we want a balance in the evolution, such as demonstrated by the e-fold form's property that $G_{\text {eff }}-1$ is a time lagged, scaled version of $\alpha_{B}(a)$.

Figure 1 shows the delay and scaling between $\alpha_{B}$ and $G_{\text {eff }}-1$ for three values of $a_{t}$. Note that $G_{\text {eff }}-1$ obeys these relations given by Eqs. (9) and (10). Thus a transition in $\alpha_{B}$ at $a_{t}=0.25$ does not give a transition in $G_{\text {eff }}$ until $a_{t, G}=0.44$, and the maximum modification of the gravitational strength is slightly over half the maximum of $\alpha_{B}$.

In addition to ensuring that deviation in the gravitational strength from general relativity can occur, and gravity remains positive, we also must ensure the stability of the model. This corresponds to the sound speed squared being nonnegative, $c_{s}^{2} \geq 0$, and we plot this as well in Fig. 1. In No Run Gravity,

$$
\alpha c_{s}^{2}=\alpha_{B}\left(1-\frac{\alpha_{B}}{2}\right)+\alpha_{B}^{\prime}-\frac{3}{2} \Omega_{m}(a) \alpha_{B}
$$

in the matter dominated era and later, where $\alpha=$ $\alpha_{K}+(3 / 2) \alpha_{B}^{2} \geq 0$, and $\Omega_{m}(a)=1-\Omega_{\mathrm{de}}(a)$ is the dimensionless matter density.

The condition $c_{s}^{2} \geq 0$ is satisfied at early times if $\alpha_{B}^{\prime} \geq$ $\alpha_{B} / 2$, corresponding to $\tau \leq 2$, i.e. the transition takes less than two e-folds. In fact, since this is an early time limit we can state this more generally than an assumption of a steplike function over a long interval. Simply put, if at early times $\alpha_{B} \sim a^{1 / \tau}$ then we require $\tau \leq 2$. That is, $\alpha_{B}$ must grow faster than $a^{1 / 2}$. In the late time limit, we require $\alpha_{B} \leq 2$, or in the e-fold case, $A \leq 2$.

Stability could be satisfied in both limits, but fail at some intermediate redshift, as seen in Fig. 1. Generally one would have to scan numerically through the model parameter space, for all redshifts, to check the model is healthy. However, again a virtue of the e-fold model is that this can be done analytically for $\tau=1 / 3$, corresponding to early time deviations $\alpha_{B} \sim a^{3}$. The solution to the boundary where $c_{s}^{2}=0$ is given by

$$
a_{t}^{3}=\frac{1}{32} \frac{\Omega_{m}}{1-\Omega_{m}}[14-A-\sqrt{15(2-A)(6-A)}] .
$$

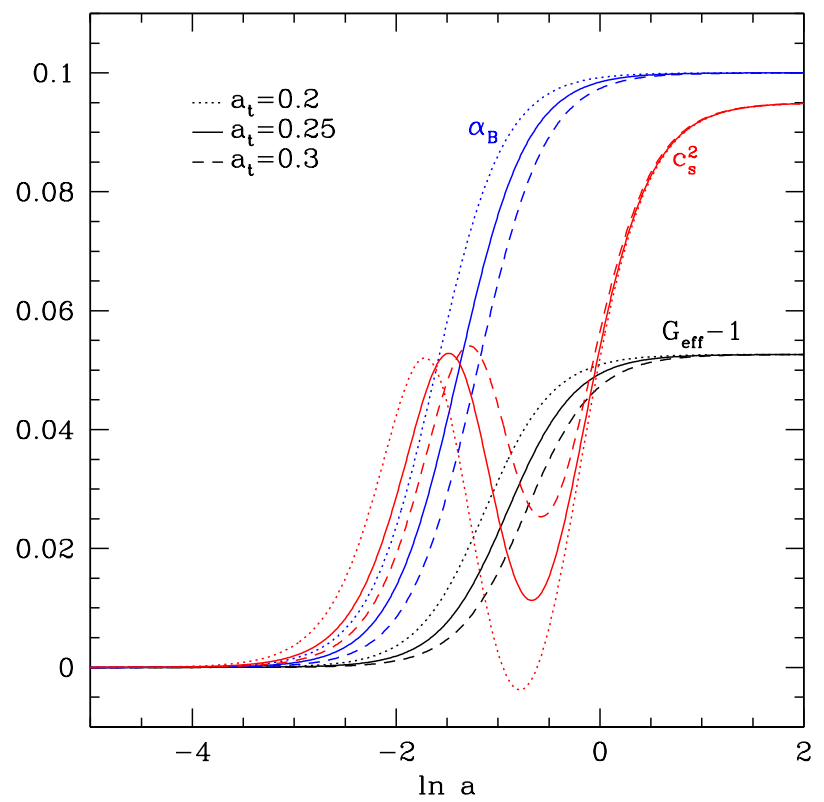

FIG. 1. An e-fold transition in the braiding property function $\alpha_{B}$ (blue curves) induces a lagged, scaled e-fold modification of the gravitational strength $G_{\text {eff }}$ (black curves) away from its general relativity value of one. Here the results are shown for three different values of the transition scale factor $a_{t}$, for fixed $A=0.1, \tau=1 / 3$. Too early a transition leads to the sound speed squared (red curves) going negative and an unstable model.

Figure 2 illustrates the constraint in the $A-a_{t}$ plane, with the unhealthy region where at some redshift $c_{s}^{2}<0$ shaded red. Interestingly, a quite good approximation to the boundary is given by a simple linear function: $a_{t}=0.198+0.135 A$. Apart from soundness, one could add observational limits, such as that gravity is no more than $10 \%$ stronger than general relativity. This would cut across the stable region of the figure at approximately $\alpha_{B, \max } \lesssim 0.2$. We consider observational constraints more rigorously in Sec. IV.

One could extend the e-fold form to multiple plateaus, either in $G_{\text {eff }}$ or $\alpha_{B}$, e.g.

$$
\alpha_{B}=\frac{A_{1}}{1+\left(a / a_{1}\right)^{-1 / \tau_{1}}}+\frac{A_{2}-A_{1}}{1+\left(a / a_{2}\right)^{-1 / \tau_{2}}},
$$

and calculate $G_{\text {eff }}$ analytically or numerically. This offers the freedom of having a further step up or step down after an intermediate phase. Indeed with $N$ steps one could approximate any desired function. However, the number of model parameters doubles with each step and anything beyond the simple case will be difficult to constrain observationally.

Furthermore, a reduction in $\alpha_{B}^{\prime}$, or even its negative value for a decreasing $\alpha_{B}^{\prime}(a)$, can turn models unstable that were stable for the single e-fold form. Moreover, a monotonically increasing $\alpha_{B}$ can still give rise to a nonmonotonic $G_{\text {eff }}$ due to the interaction of $\alpha_{B}$ and $\alpha_{B}^{\prime}$ in 


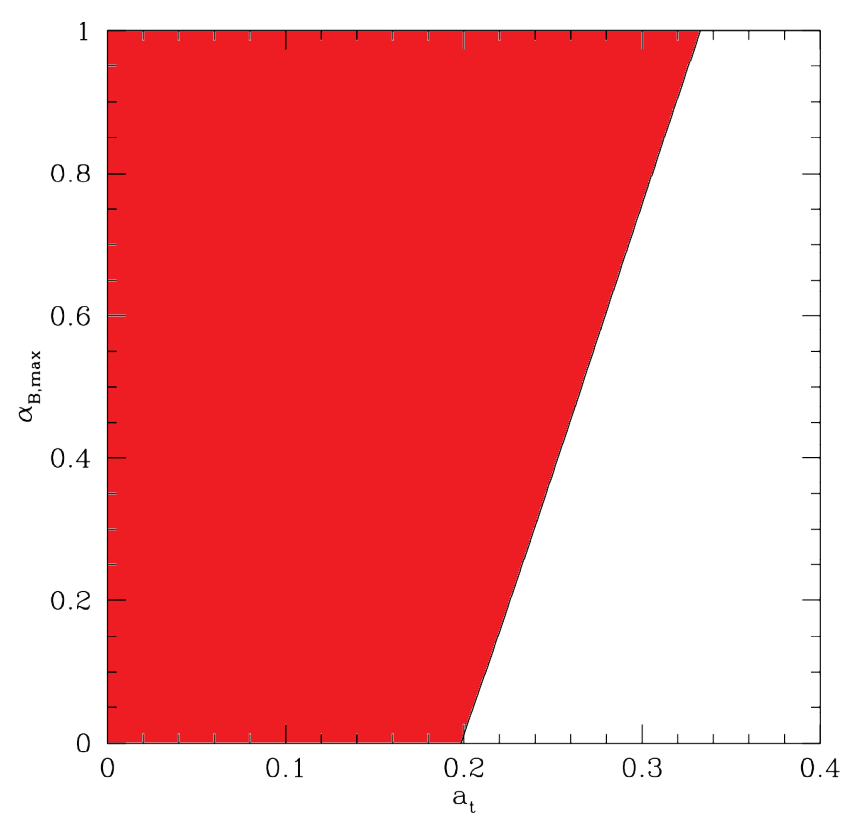

FIG. 2. The e-fold model parameter space of the maximum amplitude $A \equiv \alpha_{B, \max }$ and transition scale factor $a_{t}$ can be divided into an unstable region (red shaded) where at some redshift $c_{s}^{2}<0$ and a healthy region (unshaded). Here $\tau=$ $1 / 3$.

Eq. (4). We demonstrate these properties in Fig. 3 for a second e-fold transition stepping either up or down.

Note that a period of decreasing $\alpha_{B}$, i.e. negative $\alpha_{B}^{\prime}$, can be dangerous, as $G_{\text {eff }}$ diverges when $\alpha_{B}^{\prime}=-\alpha_{B}(1-$ $\left.\alpha_{B} / 2\right)$. A divergence necessarily means that $c_{s}^{2}<0$, as by Eq. (11) the value of $c_{s}^{2}$ is the denominator of $G_{\text {eff }}$ minus $(3 / 2) \Omega_{m}(a) \alpha_{B}$. Conversely, one can have $c_{s}^{2}<0$ without $G_{\text {eff }}$ being far from one. However, a decreasing $\alpha_{B}$ can enhance $G_{\text {eff }}$ without causing it to diverge.

Figure 4 shows the regions of instability and of high gravity $\left(G_{\text {eff }}>1.1\right.$ for $\left.a \leq 1\right)$, scanning through the amplitude-transition scale parameter space of the two efold transition model. When the amplitude of the second transition is below that of the first, i.e. $\alpha_{B}$ decreases, then instability can easily arise, as seen by the lower shaded regions with $A_{2}$ below $A_{1}=0.1$. For strong increases in amplitude at early enough times, as in the upper shaded regions, this adds to the first transition amplitude and effectively moves the model into the unstable region of Fig. 2. Even if the model stays stable, the increase in amplitude can cause $G_{\text {eff }}$ to become stronger than may be observationally viable.

Finally, let us note several model independent properties. From Eq. (5), we see that $G_{\text {eff }} \geq 1$ for viable models, independent of the form adopted for $\alpha_{B}(a)$. In order to obtain $G_{\text {eff }}<1$, the denominator would have to pass through zero, which gives not only a period of divergent gravity, but instability. Thus, No Run Gravity is in sharp contrast to No Slip Gravity by having stronger
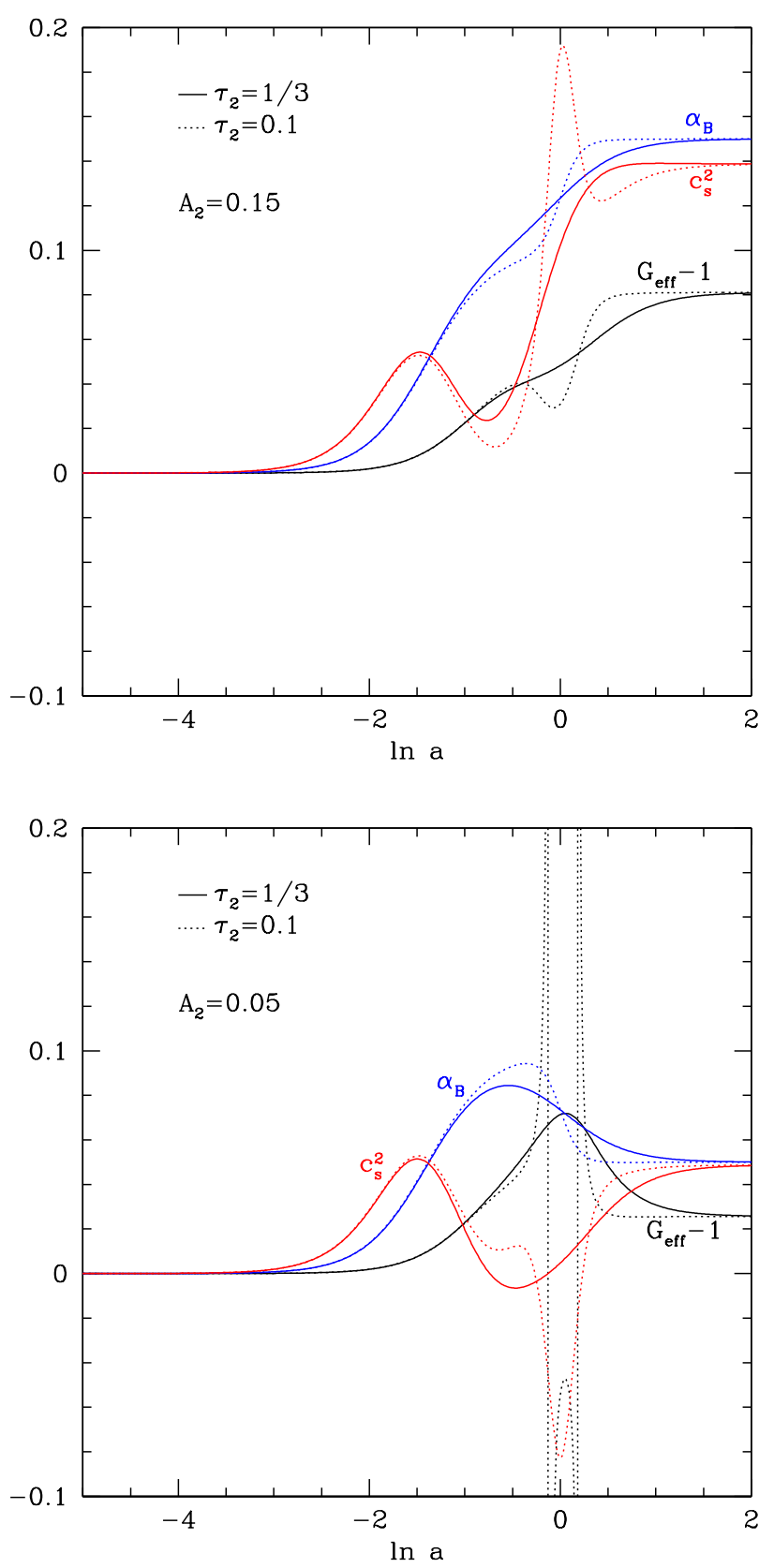

FIG. 3. For a more complicated dependence of $\alpha_{B}(a)$ the behavior of $G_{\text {eff }}(a)$ and $c_{s}^{2}(a)$ can be nonmonotonic, negative, or divergent. Here $\alpha_{B}(a)$ is given by two e-fold transitions, with $A_{1}=0.1, a_{1}=0.25, \tau=1 / 3$ as before but $a_{2}=1$ and $\tau_{2}=1 / 3$ (solid curves) or 0.1 (dotted). The top panel shows a step up, with $A_{2}=A_{1}+0.05$; the bottom panel shows a step down, with $A_{2}=A_{1}-0.05$.

gravity rather than weaker gravity than general relativity.

In fact, we can quantify the upper limit on $G_{\text {eff }}$ in a model independent manner. Rewriting the stability 


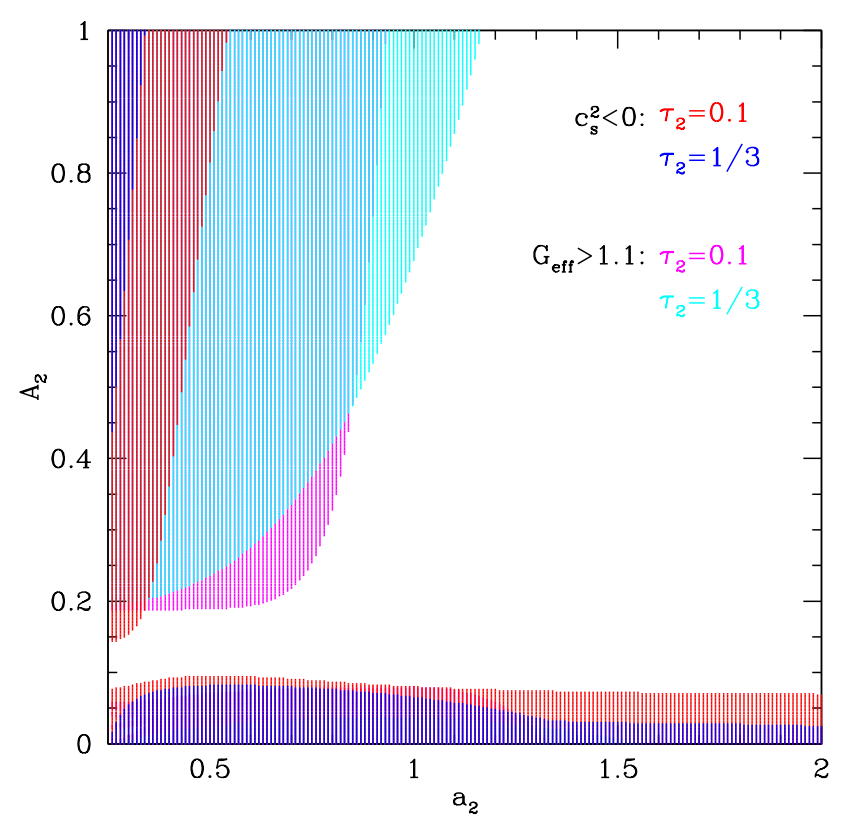

FIG. 4. The instability and high gravity regions of the parameter space are shown for the two e-fold transition form of $\alpha_{B}(a)$. The first transition has $A_{1}=0.1, a_{1}=0.25, \tau=$ $1 / 3$ as before and the second has $\tau_{2}=1 / 3$ (red or magenta) or 0.1 (blue or cyan). Regions in the $a_{2}-A_{2}$ plane are shaded if they are unstable with $c_{s}^{2}<0$ (red/blue) or have high gravity, $G_{\text {eff }}>1.1$ for $a \leq 1$ (magenta/cyan). For the lower shaded region the high gravity region lies wholly within the unstable region and is not shown.

condition $c_{s}^{2} \geq 0$ using Eq. (11) as

$$
\alpha_{B}\left(1-\frac{\alpha_{B}}{2}\right)+\alpha_{B}^{\prime} \geq \frac{3}{2} \Omega_{m}(a) \alpha_{B},
$$

and inserting this in the denominator of $G_{\text {eff }}$ gives

$$
G_{\mathrm{eff}}-1 \leq \frac{\alpha_{B}(a)}{3 \Omega_{m}(a)}
$$

Thus, stable models will have gravitational strength reasonably close to general relativity for modest $\alpha_{B}$. [Note that this bound is necessary but not sufficient; at late times when $\Omega_{m}(a) \rightarrow 0$, then $G_{\text {eff }}-1 \rightarrow \alpha_{B} /\left(2-\alpha_{B}\right)$.]

The gravitational strength can be related to the gravitational growth index $\gamma$ [19], where the growth rate $f \equiv \Omega_{m}(a)^{\gamma}$. At early times, $G_{\text {eff }} \approx 1+\mathcal{O}\left(a^{1 / \tau}\right)$ and so this corresponds to the $p=1 / \tau>0$ case in [20]. This gives $\gamma$ at early times less than the general relativity value, a characteristic of enhanced growth. At late times, $G_{\text {eff }} \rightarrow$ const, corresponding to the $p=0$ case (both values of $p$ are independent of the form of the transition, being asymptotic values). We verify numerically that, as in [20], the late time behavior of $\gamma(a)$ follows the asymptotic evolutionary form of general relativity.

\section{NEXT GENERATION CONSTRAINTS}

While the more complicated forms $\alpha_{B}(a)$ can give viable results, avoiding regions of instability and excessively high gravity, they also significantly increase the number of parameters. Therefore in this section dedicated to observational constraints we will stay with the single transition case.

We focus on the growth rate (times the amplitude) of the matter density perturbations, $f \sigma_{8}$, as the main observational constraint. While we will use the full numerical solutions of the growth equation, note that No Run Gravity is another example of a gravity theory successfully approximated by the two bin parametrization of $G_{\text {matter }}$ [21]. Indeed, $f \sigma_{8}$ is accurately reconstructed over the observational redshift range to within $0.1 \%$ of the numerical solution.

The theory changes the redshift space distortion observable $f \sigma_{8}(a)$ from $\Lambda \mathrm{CDM}$ within general relativity, but such changes can be made by changing the background expansion as well. This is why it is useful to examine both the expansion history and the growth history in a conjoined manner. We study No Run Gravity with a $\Lambda \mathrm{CDM}$ background and compare it to general relativity with other backgrounds, by means of a conjoined history diagram of the expansion rate $H / H_{0}$ and the growth rate $f \sigma_{8}$, as proposed in [22]. (Note [22] examined changes in both the matter density $\Omega_{m}$ and the dark energy equation of state parameter $w$, concluding that modified gravity had signatures in such a diagram distinct from either of them.)

Figure 5 illustrates the results, for our fiducial model of $\Omega_{m}=0.3, A=0.1, a_{t}=0.25, \tau=1 / 3$. Time, or scale factor, runs along each curve from early times at the top to today at the bottom. At a given expansion rate, the growth observable is greater than the general relativity curve with the same background. The bump, or "nose", of the modified gravity curve cuts across the general relativity models of various backgrounds, giving a distinctive signature.

To understand the level of constraints that may be placed on the modified gravity model, we consider upcoming measurements of the growth observable from redshift space distortion data of the Dark Energy Spectroscopic Instrument (DESI [23]). This will measure $f \sigma_{8}$ over $z=0.05-1.85$ with precisions approaching percent level. We follow the projections given by [24] of data precision in 18 redshift bins within this range, using only linear scales out to $k_{\max }=0.1 \mathrm{~h} / \mathrm{Mpc}$. To represent other data, such as cosmic microwave background measurements, we include a Gaussian prior of 0.01 on $\Omega_{m}$.

The results from a Fisher information analysis for the joint confidence contour of the modified gravity maximum amplitude and matter density are shown in Fig. 6 . This uses the single e-fold transition model with a fiducial value of $\Omega_{m}=0.3, A=0.1, a_{t}=0.25$, and $\tau=1 / 3$. We fix $\tau$ to the fiducial value, representing the early time behavior $\alpha_{B} \sim a^{3}$ as seems reasonable, and fix $a_{t}=0.25$, 


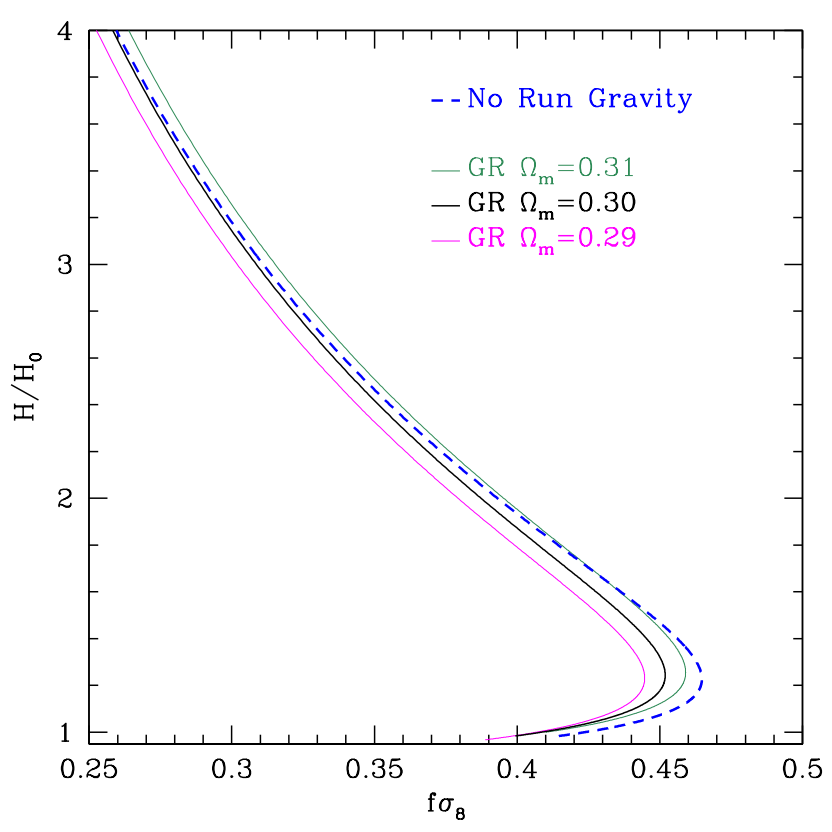

FIG. 5. No Run Gravity can be distinguished from general relativity when considering both growth history and expansion history. Note the growth $f \sigma_{8}$ is enhanced at a given expansion rate $H$ over the general relativity model with the same matter density $\left(\Omega_{m}=0.3\right)$. The conjoined history behavior is also distinct from a change in the cosmological model within general relativity.

again somewhat reasonable in order to give a stable theory, yet one that may be connected to the onset of cosmic acceleration (recall $a_{t, G}=0.44$ or $z_{t, G}=1.3$ ). If we instead marginalize over these parameters then constraints are uninterestingly weak. We emphasize that we seek to explore indications of sensitivity, not carry out a detailed likelihood fit.

The constraint on the modified gravity maximum amplitude is $\sigma(A)=0.060$, i.e. a $1.7 \sigma$ distinction from general relativity in our fiducial case. If robust density perturbation theory allows use of the measurements to $k_{\max }=0.2 h / \mathrm{Mpc}$, then the constraint tightens to $\sigma(A)=0.049$. If we had blithely extended the early time behavior $\alpha_{B} \propto \Omega_{\mathrm{de}}(a)$ to the late time observational epoch, the fiducial constraints blow up to $\sigma(A)=0.8-$ this demonstrates the danger of such an assumption as the transition occurs so late $\left(a_{t, G}=1.32\right.$ or $\left.z_{t, G}=-0.24\right)$ that even a large modification cannot be seen in the data. Thus use of such a model could lead to the conclusion that general relativity is correct, even when the true theory is quite far from general relativity.

Further data has the potential to improve our tests of modified gravity. Peculiar velocity surveys can probe growth at low redshifts $z \lesssim 0.3[25-27]$ where galaxy clustering surveys have limited sampling and yet where the effects of gravitational modifications may be strongest. The surveys may use galaxy fundamental plane distances

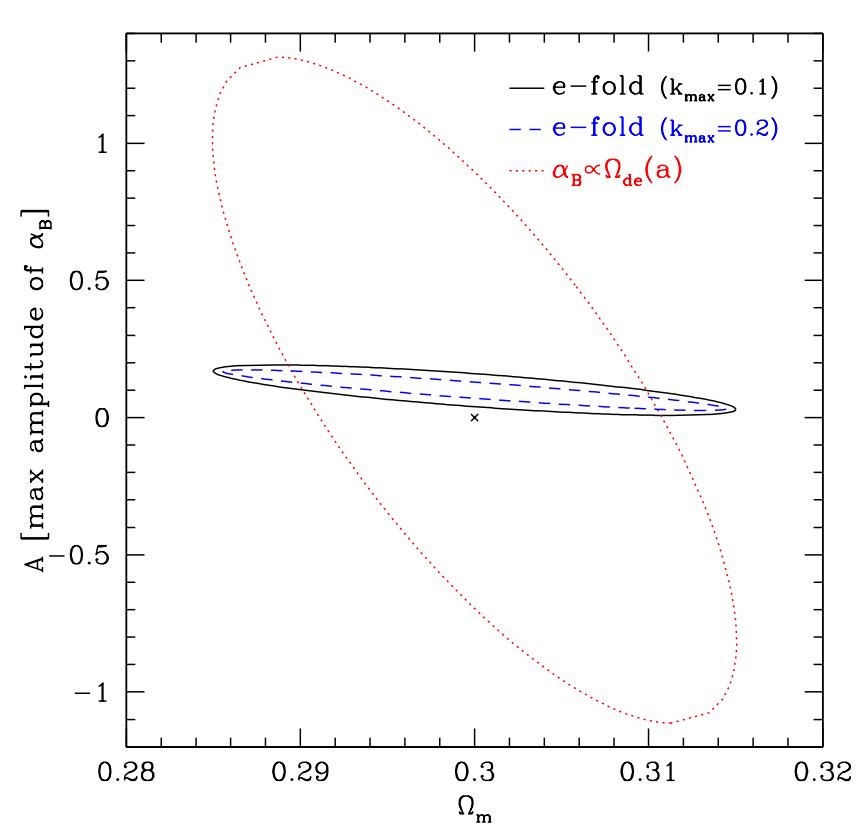

FIG. 6. The joint $68 \%$ confidence level contour for No Run Gravity is plotted in the maximum amplitude $A$ vs matter density $\Omega_{m}$ plane, for DESI projected measurements of the redshift space distortion observable $f \sigma_{8}$. The solid black contour uses $k_{\max }=0.1 \mathrm{~h} / \mathrm{Mpc}$; the dashed blue has $k_{\max }=0.2 h / \mathrm{Mpc}$. The dotted red contour shows the unrecommended $\alpha_{B} \propto \Omega_{\mathrm{de}}(a)$ model rather than the free e-fold model. The $\mathrm{x}$ marks the general relativity case with the same expansion history.

(e.g. from Taipan+WALLABY [28] or an adapted DESI Bright Galaxy survey) or supernova distances (e.g. from LSST) to obtain peculiar velocities.

\section{CONCLUSIONS}

Upcoming data will have substantial leverage on testing gravity on cosmic scales. The combination of measurements in galaxy clustering, velocities, and lensing, CMB lensing and B-mode polarization, and gravitational wave standard sirens will probe the tensor sector of gravity, gravitational slip, and the properties of structure growth and light deflection. Signatures of deviations from general relativity in any of them would be revolutionary.

However, structure growth and light deflection can also be affected by different models within general relativity. If no significant deviation is seen in gravitational wave propagation or gravitational slip, we may be tempted to say gravity is simply described by general relativity. Here we emphasize that this is not a necessary conclusion. We present No Run Gravity as a minimal modification benchmark - one where gravitational waves and gravitational slip behave as in general relativity, but there are 
signatures distinct from general relativity. These go beyond a modification to the expansion history, i.e. $w(z)$, and beyond simple scalar fields with an extra degree of freedom in the scalar sound speed.

In terms of property functions, No Run Gravity effectively has a single free function, the braiding $\alpha_{B}(a)$. We show how this maps directly to the gravitational strengths for cosmic growth and light deflection, and that these two are both the same as each other and different from general relativity. The simple e-fold form is particularly attractive since then $G_{\text {eff }}(a)$ is exactly a shifted, scaled form of $\alpha_{B}(a)$.

We quantified the gravitational strength and sound speed evolution, and analyzed the stability regions for a sound theory (indeed these are analytic for a particular model), as well as studying the gravitational growth index $\gamma$ for cosmic growth, related to $G_{\text {eff }}$. Several relations for the stability and maximum gravitational strength can be written in a model independent manner - for example for stable No Run Gravity $\left(G_{\text {eff }}-1\right) \leq \alpha_{B} /\left[3 \Omega_{m}(a)\right]$.
In addition we explored how the conjoined use of expansion and growth data could reveal modified gravity signatures, and carried out an initial Fisher information sensitivity analysis for how forthcoming DESI data on the growth rate $f \sigma_{8}$ from redshift space distortion measurements could constrain the gravitational strength $G_{\text {eff }}$. No Run Gravity can provide a benchmark for distinguishing minimal modification signatures from general relativity, showing the science reach of next generation surveys even when the more dramatic signatures may not be found.

\section{ACKNOWLEDGMENTS}

This work is supported in part by the Energetic Cosmos Laboratory and by the U.S. Department of Energy, Office of Science, Office of High Energy Physics, under Award DE-SC-0007867 and contract no. DE-AC02$05 \mathrm{CH} 11231$.
[1] M. Lagos, M. Fishbach, P. Landry, D.E. Holz, Standard sirens with a running Planck mass, arXiv:1901.03321

[2] Simons Observatory Collaboration, The Simons Observatory: Science goals and forecasts, arXiv:1808.07445

[3] B.P. Abbott et al., Gravitational Waves and GammaRays from a Binary Neutron Star Merger: GW170817 and GRB 170817A, Astrophys. J. Lett. 848, L13 (2017) [arXiv: 1710.05834]

[4] L. Lombriser, A. Taylor, Breaking a Dark Degeneracy with Gravitational Waves, JCAP 1603, 031 (2016) [arXiv:1509.08458]

[5] A. Nishizawa, Generalized framework for testing gravity with gravitational-wave propagation. I. Formulation, Phys. Rev. D 97, 104037 (2018) [arXiv:1710.04825]

[6] S. Arai and A. Nishizawa, Generalized framework for testing gravity with gravitational-wave propagation. II. Constraints on Horndeski theory, Phys. Rev. D 97, 104038 (2108) [arXiv:1711.03776]

[7] E.V. Linder, No Slip Gravity, JCAP 1803, 005 (2018) [arXiv:1801.01503]

[8] M. Ishak, Testing General Relativity in Cosmology, Living Rev. Relativ. 22, 1 (2019) [arXiv:1806.10122]

[9] P.G. Ferreira, Cosmological Tests of Gravity, arXiv:1902.10503

[10] C. Armendariz-Picon, V. Mukhanov, P. Steinhardt, Dynamical Solution to the Problem of a Small Cosmological Constant and Late-Time Cosmic Acceleration, Phys. Rev. Lett. 85, 4438 (2000) [arXiv:astro-ph/0004134]

[11] T. Chiba, T. Okabe, M. Yamaguchi, Kinetically Driven Quintessence, Phys. Rev. D 62, 023511 (2000) [arXiv:astro-ph/9912463]

[12] E. Bellini, I. Sawicki, Maximal freedom at minimum cost: linear large-scale structure in general modifications of gravity, JCAP 1407, 050 (2014) [arXiv:1404.3713]

[13] L. Amendola, M. Kunz, I.D. Saltas, I. Sawicki, The fate of large-scale structure in modified gravity after GW170817 and GRB170817A, Phys. Rev. Lett. 120,
131101 (2018) [arXiv:1711.04825]

[14] M. Brush, E.V. Linder, M. Zumalacárregui, No Slip CMB, JCAP 1901, 029 (2019) [arXiv:1810.12337]

[15] C. Deffayet, O. Pujolas, I. Sawicki, A. Vikman, Imperfect Dark Energy from Kinetic Gravity Braiding, JCAP 1010, 026 (2010) [arXiv:1008.0048]

[16] T. Kobayashi, M. Yamaguchi, J. Yokoyama, G-inflation: inflation driven by the Galileon field, Phys. Rev. Lett. 105, 231302 (2010) [arXiv:1008.0603]

[17] S. Appleby, E.V. Linder, The Well-Tempered Cosmological Constant, JCAP 1807, 034 (2018) [arXiv:1805.00470]

[18] E.V. Linder, G. Sengör, S. Watson, Is the Effective Field Theory of Dark Energy Effective?, JCAP 1605, 053 (2016) [arXiv:1512.06180]

[19] E.V. Linder, Cosmic Growth History and Expansion History, Phys. Rev. D 72, 043529 (2005) [arXiv:astro-ph/0507263]

[20] E.V. Linder, D. Polarski, The End of Cosmic Growth, Phys. Rev. D 99, 023503 (2019) [arXiv:1810.10547]

[21] M. Denissenya, E.V. Linder, Subpercent Accurate Fitting of Modified Gravity Growth, JCAP 1711, 052 (2017) [arXiv:1709.08709]

[22] E.V. Linder, Cosmic Growth and Expansion Conjoined, Astropart. Phys. 86, 41 (2017) [arXiv:1610.05321]

[23] DESI Collaboration, The DESI Experiment Part II: Instrument Design, arXiv:1611.000037

[24] DESI Collaboration, The DESI Experiment Part I: Science,Targeting, and Survey Design, arXiv:1611.00036

[25] C. Adams, C. Blake, Improving constraints on the growth rate of structure by modelling the density-velocity crosscorrelation in the 6dF Galaxy Survey, MNRAS 471, 839 (2017) [arXiv:1706.05205]

[26] C. Howlett, L. Staveley-Smith, C. Blake, Cosmological Forecasts for Combined and Next Generation Peculiar Velocity Surveys, MNRAS 464, 2517 (2017) [arXiv:1609.08247] 
[27] C. Howlett, A.S.G. Robotham, C.D.P. Lagos, A.G. Kim, Measuring the growth rate of structure with Type IA Supernovae from LSST, ApJ 847, 128 (2017) [arXiv:1708.08236]
[28] E. da Cunha et al, The Taipan Galaxy Survey: Scientific Goals and Observing Strategy, PASA 34, 47 (2017) [arXiv:1706.01246] 